Frank Duschek*, Lea Fellner, Florian Gebert, Karin Grünewald, Anja Köhntopp, Marian Kraus, Peter Mahnke, Carsten Pargmann, Herbert Tomaso and Arne Walter

\title{
Standoff detection and classification of bacteria by multispectral laser-induced fluorescence
}

DOI 10.1515/aot-2016-0066

Received December 2, 2016; accepted February 6, 2017

Abstract: Biological hazardous substances such as certain fungi and bacteria represent a high risk for the broad public if fallen into wrong hands. Incidents based on bio-agents are commonly considered to have unpredictable and complex consequences for first responders and people. The impact of such an event can be minimized by an early and fast detection of hazards. The presented approach is based on optical standoff detection applying laser-induced fluorescence (LIF) on bacteria. The LIF bio-detector has been designed for outdoor operation at standoff distances from $20 \mathrm{~m}$ up to more than $100 \mathrm{~m}$. The detector acquires LIF spectral data for two different excitation wavelengths (280 and $355 \mathrm{~nm}$ ) which can be used to classify suspicious samples. A correlation analysis and spectral classification by a decision tree is used to discriminate between the measured samples. In order to demonstrate the capabilities of the system, suspensions of the low-risk and non-pathogenic bacteria Bacillus thuringiensis, Bacillus atrophaeus, Bacillus subtilis, Brevibacillus brevis, Micrococcus luteus, Oligella urethralis, Paenibacillus polymyxa and Escherichia coli (K12) have been investigated with the system, resulting in a discrimination accuracy of about $90 \%$.

Keywords: bacteria; biological agents; classification; laser-induced fluorescence (LIF); standoff detection.

*Corresponding author: Frank Duschek, German Aerospace Center, Institute of Technical Physics, Langer Grund, Lampoldshausen, 74239 Hardthausen, Germany, e-mail: frank.duschek@dlr.de Lea Fellner, Florian Gebert, Karin Grünewald, Marian Kraus, Carsten Pargmann and Arne Walter: German Aerospace Center, Institute of Technical Physics, Langer Grund, Lampoldshausen, 74239 Hardthausen, Germany

Anja Köhntopp and Peter Mahnke: German Aerospace Center, Institute of Technical Physics, 70569 Stuttgart, Germany Herbert Tomaso: Friedrich-Loeffler-Institute, Institute of Bacterial Infections and Zoonoses, 07743 Jena, Germany

www.degruyter.com/aot
OCIS codes: 160.1435; 280.1415; 300.2530; 280.0280; 000.3860 .

\section{Introduction}

Hazardous bacteria represent a major threat to modern societies if released into the environment. Aerosols of bacteria can occur naturally when infected animals or humans spread a pathogen, but they can also be released by accidents and as a terrorist attack. Once people are infected, bacteria may easily spread aided by the fact that their detrimental effects can occur with a certain time delay. Therefore, fast and reliable detection and identification of bacteria is essential for the containment and the decontamination of affected areas and people. Commonly used methods for bacterial identification are mass spectrometry, polymerase chain reaction, DNA sequencing and flow cytometry [1], all of which are employed in the form of point sensors. These methods are highly sensitive and able to identify bacteria very accurately. At the same time they require direct contact with a sample, can only be used to cover small areas, and are not suitable for real-time detection or tracking of moving aerosol sources. Optical, i.e. laser-based methods on the other hand are capable of monitoring large areas in real time and from a safe distance. Here, additional challenges lie in compensating the atmospheric interferences, i.e. incident sunlight, light scattering and absorption from air, and airborne particles (pollen, dust, raindrops) and other weather-related phenomena. Furthermore, in inhabited areas the used laser radiation must be eye-safe. Biological samples contain a large number of fluorophores. Therefore, laser-induced fluorescence (LIF) yields high signal intensities at relatively low excitation energies [2, 3]. As fluorescence spectra usually show very few distinct features, the combination with sophisticated data analysis is necessary to increase specificity and sensitivity. The use of several excitation wavelengths has been shown to strongly enhance the capabilities for identification/classification [4]. Additionally, the fluorescence lifetime of bacteria has been used to characterize a small set of them $[5,6]$. 
While spectroscopy on chemical solutions is rather straightforward due to well-defined structures of chemical agents, bacteria represent a more complex problem. They greatly differ in size and shape, and sometimes they form clusters, films or chains. Depending on the environmental conditions bacteria can exist in different states, i.e. vegetative cells, endospores or dead. As their chemical composition is very complex, a number of fluorophores contribute to the observed overall fluorescence. For Escherichia coli 15 different fluorophores have been identified [7]. After excitation in the UV $(280 \mathrm{~nm})$ the observed fluorescence is dominated by the amino acid tryptophan and shows smaller contribution from tyrosine, while at an excitation wavelength of $355 \mathrm{~nm}$, nicotinamide adenine dinucleotide $(\mathrm{NADH})$ and flavins are the main contributors.

The discrimination of different bacteria by their intrinsic fluorescence has been demonstrated under laboratory conditions $[8,9]$. Furthermore, several approaches towards a standoff detection system based on multiple-wavelength LIF have been presented. Pan et al. [10, 11] developed an instrument that detects LIF from excitation wavelengths of 263 and $351 \mathrm{~nm}$ and scattering of single aerosol particles. The instrument is capable of classifying airborne pollen and fungal particles. The US Naval Research Laboratory presented a similar approach, in which the dual excitation wavelength fluorescence of single aerosol particles is recorded in four different wavelength channels [12]. Under laboratory conditions, the discrimination of different biological and non-biological aerosol particles by LIF techniques was successfully demonstrated [13]. The Swedish Defence Research Agency (FOI) developed a light detection and ranging (LIDAR) UV-LIF system for standoff aerosol detection, which has been field-tested for the classification of different biological particles and interferants [14]. The LIDAR technique has also been used by others, for example, research groups from Italy [15], India [16] and Canada [17, 18]. The Canadian SINBAHD system has gone through several field tests concerning the recording of spectral signatures of different bacteria, toxins and virus simulants under cross-wind conditions at standoff distances up to the kilometer range. Recently, a scanning IR-LIDAR has been developed that monitors the surroundings for aerosol clouds and triggers a staring UV spectrometric LIF LIDAR to spectrally classify the biological components in the cloud. This system is combined with point sensors using particle size-fluorescence distribution for identification to achieve higher sensitivity and precision [19]. A lot of progress has been made in the last few decades, but currently there is no system offering the necessary speed, sensitivity, robustness and reliability for everyday application.
In cooperation with LDI Innovations (Estonia), we presented a setup for dual-excitation wavelength $(280 \mathrm{~nm}, 355 \mathrm{~nm})$ LIF detection and classification of different bioorganic substances [20, 21]. The online classifier used therein relies on spectral feature extraction and selection and has been trained with a basic set of experimental spectra by bootstrap aggregation and a decision tree classification. The results showed the discrimination of a variety of bioorganic substances into four classes: plants, oils, chemicals and bacteria [3, 22].

The next step is to investigate the ability to discriminate different bacterial species with optical methods. Here, we present the experimental results for a small set of eight different low-risk bacteria. Multispectral LIF spectra were recorded at a standoff distance of $22 \mathrm{~m}$ at excitation wavelengths of 280 and $355 \mathrm{~nm}$. Within this predetermined group of bacteria, we demonstrate the discrimination of samples containing different genera as well as closely related bacteria by their LIF signature.

\section{Experimental section}

\subsection{Experimental setup}

The standoff LIF detection system used in this study is operated on an outdoor optical test range at the German Aerospace Center, Lampoldshausen. The laser and detection system is operated indoors, whereas the target is positioned outdoors. Experiments can be carried out with both generated aerosols and liquids in a cuvette. All data shown here were measured for suspensions at a distance of $22 \mathrm{~m}$ at both excitation wavelengths of 280 and $355 \mathrm{~nm}$.

The experimental setup was designed as a generic system to be used not only for LIF but also for Raman and laser-induced breakdown spectroscopy, which require higher spectral resolutions. As described in Section 4, a more dedicated LIF detection system with compact detection optics and lower spectral resolution will be presented in future work.

2.1.1 Optics: The schematic setup of the detection system is shown in Figure 1. A frequency tripled Nd:YAG laser Spitlight 600-10 (InnoLas $\mathrm{GmbH}$, Krailing, Germany) supplies pulses of 7 ns pulse length at $355 \mathrm{~nm}$ with a repetition rate of $10 \mathrm{~Hz}$. Each second pulse is frequency converted to $280 \mathrm{~nm}$, resulting in an effective repetition rate of $5 \mathrm{~Hz}$ with pulse energies of about $10 \mathrm{~mJ}$ for each wavelength. For the generation of the radiation at $280 \mathrm{~nm}$ an optical parametric oscillator (OPO) setup is chosen. The design closely follows the ideas of an OPO with an additional intracavity sum frequency mixing presented and patented by Fix et al. [23-25]. The OPO setup is idler resonant for $1325 \mathrm{~nm}$ and pumped with $532 \mathrm{~nm}$ pulses from the frequency doubled Nd:YAG laser. Sum frequency mixing the $1325 \mathrm{~nm}$ with the third harmonic of the pump laser generates the radiation at $280 \mathrm{~nm}$ required to induce fluorescence of tryptophane fluorophores. The switching between the 355 and $280 \mathrm{~nm}$ radiation is performed by a synchronized intracavity shutter. Both beams are guided onto the target at a distance 


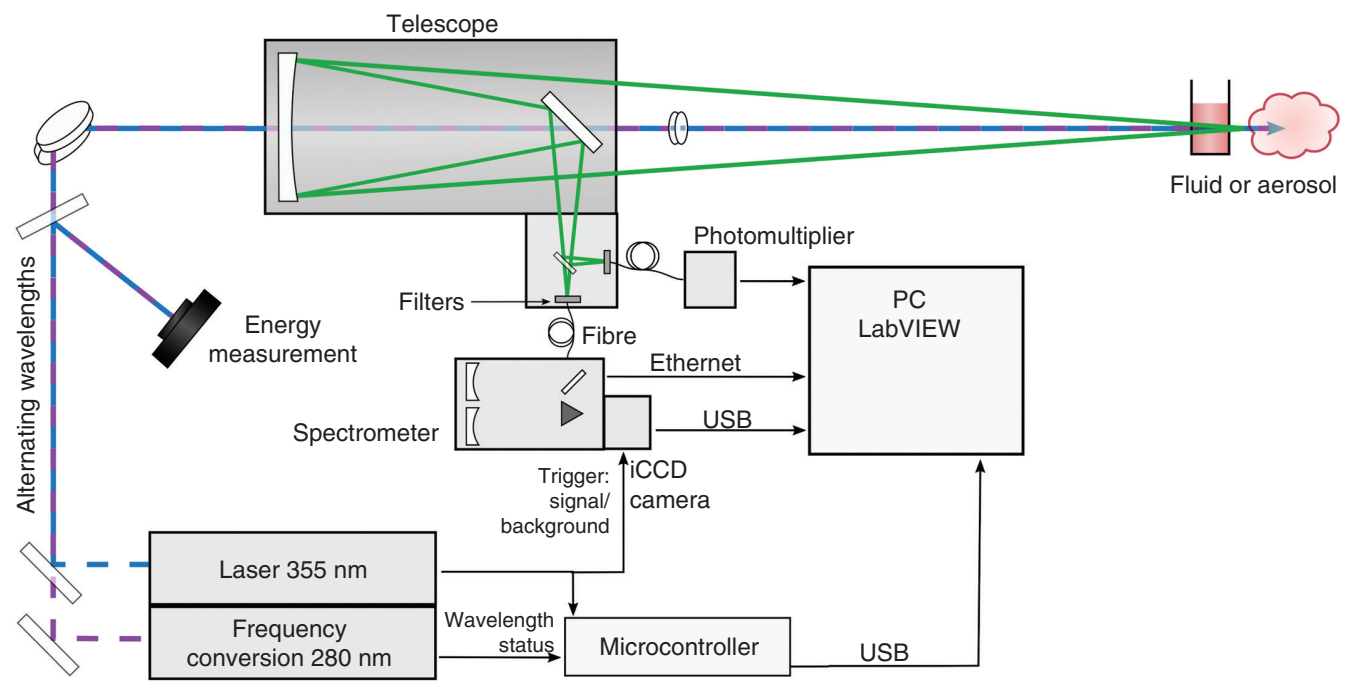

Figure 1: Schematic draft of the experimental setup. Laser pulses with alternating wavelengths of $355 \mathrm{~nm}$ (blue) and $280 \mathrm{~nm}$ (purple) are emitted. The detection path is shown in green.

of $22 \mathrm{~m}$. According to European standard (EN 60825-1) the maximum permissible exposure for UV radiation amounts to $30 \mathrm{~J} / \mathrm{m}^{2}$. In order to work with eye-safe energy densities, the beam radius of the laser radiation is enlarged to be wider than $10 \mathrm{~mm}$. The isotropically emitted fluorescence light is collected by a Newton telescope NA16 (Alluna Optics, Bobingen, Germany) with an optical diameter of $400 \mathrm{~mm}$. The collected light is split, spectrally filtered to suppress the laser lines and coupled into optical fibers. One fraction is detected by a photomultiplier tube (PMT) H10721-210 (Hamamatsu Photonics K.K., Hamamatsu City, Japan) providing information on the overall time behavior of the fluorescence signal considering the whole spectrum. The integrated signal of the PMT represents the signal strength of each spectrum and can be used for later normalization. The other fraction is analyzed by a spectrometer Acton SpectraPro SP2500 (Princeton Instruments, Trenton, NJ, USA) with a resolution of $0.42 \mathrm{~nm}$ and a spectral range of 300-600 $\mathrm{nm}$ and is captured by a gated, intensified CCD camera PI-MAX 3 (Princeton Instruments, Trenton, NJ, USA).

2.1.2 Electronics and data acquisition: The laser acts as a master trigger for the whole system. Each trigger pulse controls the capturing gate of the iCCD camera to record the fluorescence response of the target. Fifty milliseconds after the laser trigger, a second trigger is generated to record an individual background spectrum. The camera, PMT and spectrometer are controlled by a computer running self-developed software. A microcontroller (Mega 2560, Arduino LLC, Scarmagno, Italy) provides proper timing and assignment of all signal sources of the measurement process, monitors the wavelength of the alternating output of the frequency conversion unit (in-house development P. Mahnke, DLR Stuttgart) and controls a shutter Beamblock (MRC Systems GmbH, Heidelberg, Germany) for proper irradiation of the samples.

The system is capable of resolving the time dependence of the fluorescence pulse by shifting the gate delay of the camera relative to several consecutive laser pulses. Nevertheless, this acquisition mode is time consuming using a $10 \mathrm{~Hz}$ laser system and therefore the additional time information is omitted here. Hence, data acquisition and data analysis are based on a fixed gate delay setting and on 960 single-shot spectra including eight substances. Each single spectrum is linked to an individual background spectrum to compensate for fluctuations in background scattering and radiation. This makes measurements less sensitive to weather conditions, which, in free atmosphere, may fluctuate during the measurement on a timescale of seconds.

\subsection{Bacterial strains: cultivation and sample preparation}

The investigated bacterial strains are Bacillus thuringiensis var. kurstaki strain HD-1 (isolated from insecticide DIPEL ES, Cheminova, Stade, Germany), Escherichia coli K12 J53 [26], Bacillus atrophaeus (DSM 7264), Bacillus subtilis (DSM 1970), Brevibacillus brevis (DSM 30), Micrococcus luteus (DSM 20030), Oligella urethralis (DSM 7531) and Paenibacillus polymyxa (DSM 36). Figure 2 shows the evolutionary relationship of these bacterial strains. Closely related bacteria are more similar in their composition than distantly related bacteria and might have more similar fluorescence spectra. The strains assigned to the Bacillales (P. polymyxa, B. brevis, B. thuringiensis, B. subtilis, $B$. atrophaeus) are endospore-forming bacteria.

All investigated bacterial strains underwent the same procedure for sample preparation. Cultivation of the bacterial species was carried out on blood agar plates (nutrient agar 1 obtained at Sifin, Berlin, Germany) supplemented with $7.5 \%$ cattle or sheep blood (Fiebig Naehrstofftechnik, Idenstein-Niederauroff/Ts., Germany) at $37^{\circ} \mathrm{C}$ for $24 \mathrm{~h}$. Colony material was harvested and suspended in phosphate buffered saline (PBS; Dulbecco's Phosphate-Buffered Saline modified, without calcium, Sigma-Aldrich, Darmstadt, Germany) and stored at $4^{\circ} \mathrm{C}$ for 6 days. The concentration of colony-forming units (CFU) per ml was determined by cultivation of $100 \mu \mathrm{L}$ serially diluted bacterial suspensions in PBS with $0.4 \%$ agar on agar plates under identical conditions. For fluorescence measurements the bacterial suspensions were diluted with PBS to $10^{6}$ and $10^{7} \mathrm{CFU}$ for $B$. brevis, $10^{8}$ and $10^{9} \mathrm{CFU}$ for $B$. thuringiensis, E. coli, B. atrophaeus, $M$. luteus and $P$. polymyxa, $10^{9}$ and $10^{10} \mathrm{CFU}$ for $O$. urethralis, and $10^{10}$ and $10^{11} \mathrm{CFU}$ for $B$. subtilis. Measurements were conducted in $3500 \mu \mathrm{L}$ cuvettes (117-QS, Hellma GmbH \& Co. KG, Müllheim, Germany), while the samples were continuously stirred with a magnetic bar and stirrer (IKA color squid, IKA-Werke GmbH \& Co. KG, Staufen, Germany). 


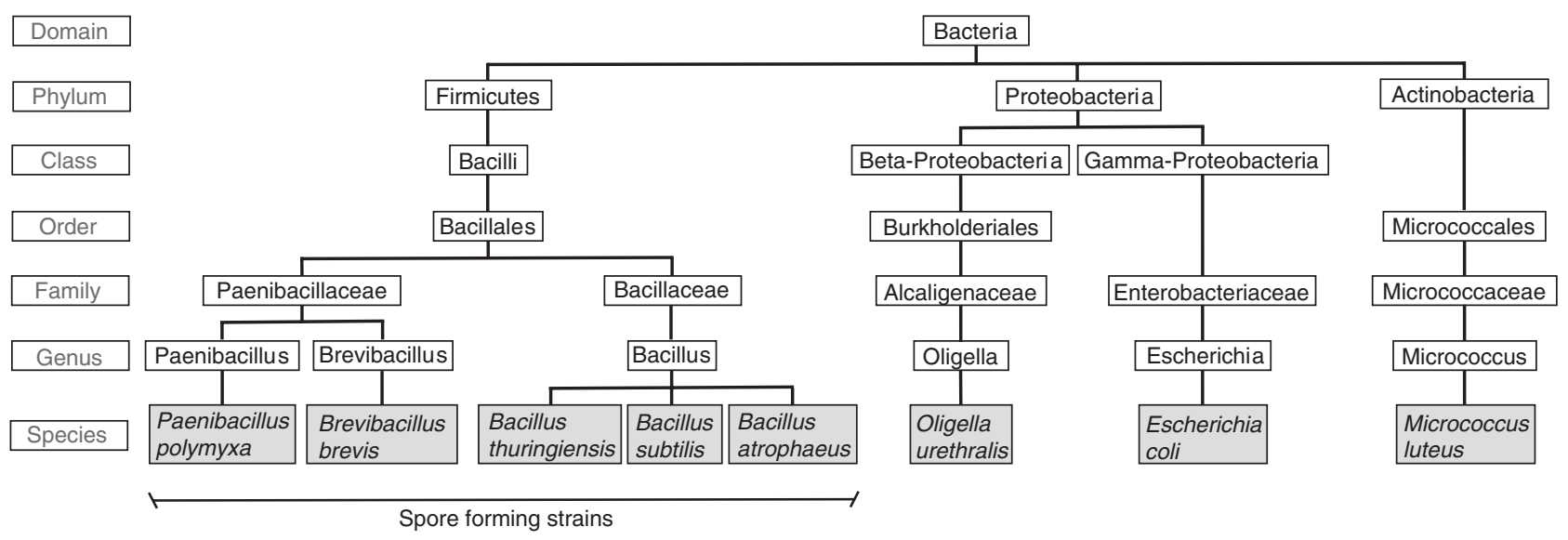

Figure 2: Evolutionary relationship of the investigated bacterial strains.

\section{Results}

\subsection{LIF spectra of bacteria}

Measurement and data acquisition settings were kept the same for all measurements to ensure comparable results. On the other hand, determination of the concentration of CFU in the samples yields results with rough accuracy only. Taking into account that some samples were provided with concentrations differing by orders of magnitude, the spectra can be compared when normalized. In Figure 3 the measured fluorescence spectra of $B$. brevis (black curve), B. thuringiensis (blue), P. polymyxa (yellow), B. atrophaeus (red), B. subtilis (green), E. coli (cyan), M. luteus (pink) and O. urethralis (olive) are presented for both excitation wavelengths. At $355 \mathrm{~nm}$ excitation, many of the spectra seem quite similar and unstructured. None of the spectra is identical over the complete spectral region between 355 and $600 \mathrm{~nm}$. For the excitation wavelength of $280 \mathrm{~nm}$, most fluorescence spectra differ strongly especially with a broad emission maximum in the range from 450 to $550 \mathrm{~nm}$. There are, however, spectra which cannot be distinguished easily: P. polymyxa, M. luteus and E. coli are nearly congruent, as well as $B$. brevis and $B$. subtilis.

\subsection{Correlation of spectral features}

In preparation of a statistical data analysis for the following classification, the correlation between the spectral data of bacteria is examined. The statistical software package $\mathrm{R}$ [27] is used to calculate the Spearman rank correlation
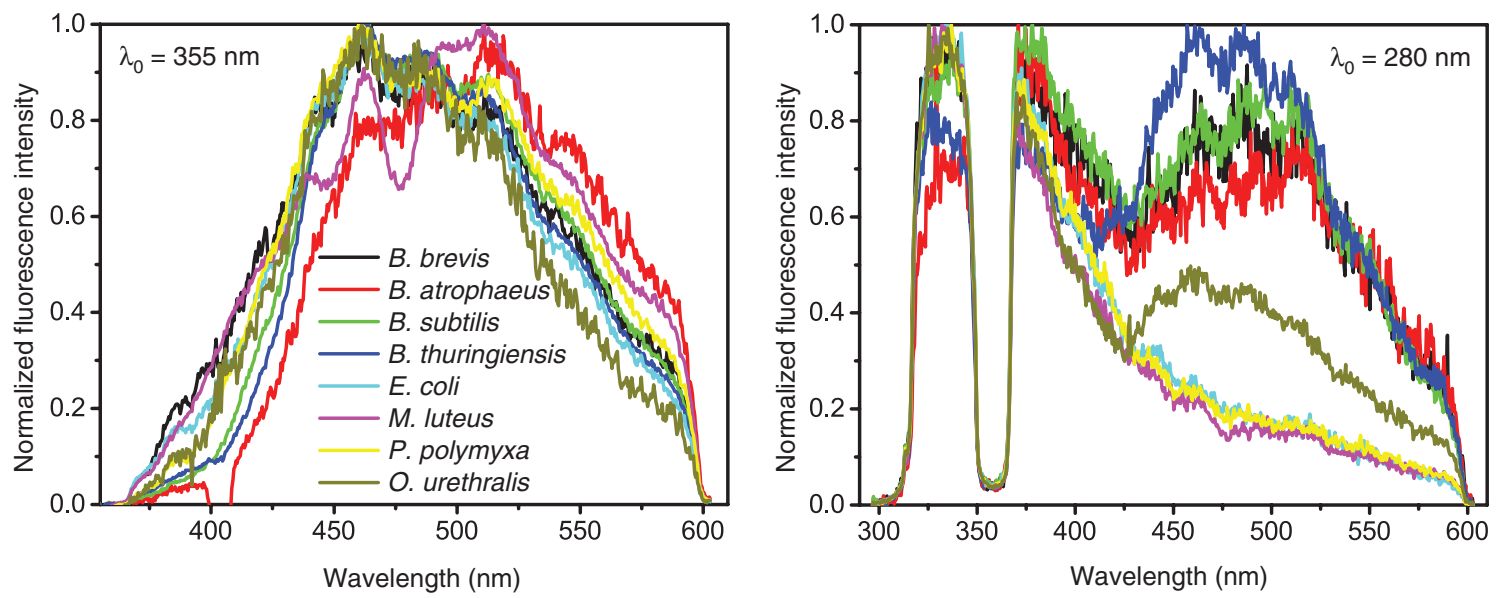

Figure 3: Normalized LIF spectra of bacteria samples excited at $\lambda_{0}=355 \mathrm{~nm}$ (left panel) and $\lambda_{0}=280 \mathrm{~nm}$ (right). Concentrations (in CFU $/ \mathrm{ml}$ ): B. brevis $10^{7}$; B. thuringiensis, P. polymyxa $10^{8}$; B. atrophaeus, B. subtilis, E. coli, M. luteus $10^{9} ;$ O. urethralis $10^{11}$. The low intensity around $355 \mathrm{~nm}$ for $280 \mathrm{~nm}$ excitation results from a filter blocking the $355 \mathrm{~nm}$ laser pulses. 
Table 1: Linear rank correlation coefficients $r_{s}$ for spectral data of pairs of bacteria.

\begin{tabular}{llllllll}
\hline B. atrophaeus & 0.97 & 0.88 & 0.95 & 0.63 & 0.60 & 0.80 & 0.63 \\
0.94 & B. subtilis & 0.92 & 0.98 & 0.68 & 0.65 & 0.85 & 0.67 \\
0.94 & 1.00 & B. thuringiensis & 0.92 & 0.42 & 0.40 & 0.70 & 0.41 \\
0.86 & 0.94 & 0.94 & B. brevis & 0.69 & 0.68 & 0.87 & 0.69 \\
0.88 & 0.97 & 0.97 & 0.89 & E. coli & 0.99 & 0.92 & 1.00 \\
0.94 & 0.96 & 0.96 & 0.95 & 0.95 & M. luteus & 0.92 & 0.99 \\
0.80 & 0.90 & 0.90 & 0.98 & 0.96 & 0.88 & 0. urethralis & 0.92 \\
0.92 & 0.99 & 0.99 & 0.98 & 0.99 & 0.97 & 0.94 & P. polymyxa \\
\hline
\end{tabular}

Lower left: $\lambda_{0}=355 \mathrm{~nm}$; upper right: $\lambda_{0}=280 \mathrm{~nm}$.

coefficients [28]. Linear correlation, described by Spearman's coefficient $r_{s}$, is often regarded as a measure for the similarity of spectral data. In short, considering a single spectrum per bacterium, the method picks one and sorts its values by size to obtain an ascending function (socalled ranking). The remaining spectra of the dataset are then arranged in the same sequence defined by the first spectrum. Finally, the coefficient describes the means of the single slopes of all spectra in relation to the slope of the considered first spectrum. The obtained values range from -1 to +1 , i.e. from indirect to direct proportionality. Results close to 0 indicate no correlation.

Table 1 shows a summary of the correlation coefficients $r_{s}$ for the 355 and $280 \mathrm{~nm}$ excited LIF spectra in the lower-left and upper-right cells, respectively. The values of the correlation coefficients $r_{s}$ are given in the lower left. Commonly, correlation is regarded as weak if $\left|r_{s}\right| \leq 0.5$, as medium if $0.5<\left|r_{s}\right| \leq 0.8$ and as strong if $\left|r_{s}\right|>0.8$. For $355 \mathrm{~nm}$ excitation there is a strong positive linear correlation between fluorescence data. All correlation coefficients are above 0.8 , with weakest $r_{s}=0.8$ for $O$. urethralis and $B$. atrophaeus. The strong correlation indicates that the excitation wavelength of $355 \mathrm{~nm}$ has limited potential for differentiation of fluorescence data. The correlation coefficients $r_{s}$ of $280 \mathrm{~nm}$ excited spectra (Table 1, upper right) show medium to weak correlation values ( $r_{s}$ from 0.4 to 0.7 ) for many pairs of bacteria samples. Especially $B$. thuringiensis correlates weakly with $E$. coli, M. luteus and P. polymyxa.

\subsection{Discrimination of bacteria spectra}

As described in Section 2.1, each recorded output file contains single-shot spectra for both excitation wavelengths. Each sample is measured 60 times in two different concentrations. Hence, an overall dataset of 960 single-shot spectra is available for training and testing the classifier. For preparation of the modeling process, the corresponding single-shot spectra of 280 and $355 \mathrm{~nm}$ are combined, and wavelength regions lacking intensity information and spikes are removed. Then $75 \%$ randomly chosen samples are taken to train the decision tree algorithm C5.0 of the software package $\mathrm{R}[29,30]$. The spectral variation between different bacteria as well as the similarities within one species gives different information for building a decision tree. So the intensities of the single CCD channels (features) are compared and those with the highest entropy define the branching. This leads to models where as many bacteria as possible are classified

Table 2: Correlation matrix of the $\mathrm{C} 5.0$ algorithm for training data.

\begin{tabular}{|c|c|c|c|c|c|c|c|c|}
\hline Classified as $\rightarrow$ & B. atrophaeus & B. brevis & B. subtilis & B. thuringiensis & E. coli & M. luteus & o. urethralis & P. polymyxa \\
\hline \multicolumn{9}{|l|}{ Actual sample } \\
\hline B. atrophaeus & 77 & & & & 1 & & & \\
\hline B. brevis & & 91 & & 1 & 1 & & & 1 \\
\hline B. subtilis & 1 & & 94 & & & & 1 & 2 \\
\hline B. thuringiensis & & & & 84 & & & 1 & \\
\hline E. coli & & 1 & 1 & 1 & 95 & 1 & & \\
\hline M. luteus & & & & & 1 & 86 & 1 & \\
\hline O. urethralis & & 2 & & & & & 83 & \\
\hline P. polymyxa & & & 1 & & 1 & 1 & & 90 \\
\hline
\end{tabular}

Samples: 720 ; trials: 1. 
Table 3: Correlation matrix of the $C 5.0$ algorithm for test data.

\begin{tabular}{|c|c|c|c|c|c|c|c|c|}
\hline Classified as $\rightarrow$ & B. atrophaeus & B. brevis & B. subtilis & B. thuringiensis & E. coli & M. luteus & o. urethralis & P. polymyxa \\
\hline \multicolumn{9}{|l|}{ Actual sample } \\
\hline B. atrophaeus & 42 & & & & & & & \\
\hline B. brevis & & 25 & & 1 & & & & \\
\hline B. subtilis & & 1 & 22 & & 1 & 1 & & 1 \\
\hline B. thuringiensis & & 1 & & 31 & & & 1 & \\
\hline E. coli & & & 1 & & 20 & & 4 & \\
\hline M. luteus & & & & 1 & & 26 & & 2 \\
\hline O. urethralis & & 1 & & & & & 28 & \\
\hline P. polymyxa & & & 1 & & & 4 & 1 & 24 \\
\hline
\end{tabular}

Samples: 240; trials: 20.

Table 4: Confusion matrix [31] of the classification model applied to the test data.

\begin{tabular}{lrrrrrrrr}
\hline & B. atrophaeus & B. brevis & B. subtilis & B. thuringiensis & E. coli & M. luteus & O. urethralis & P. polymyxa \\
\hline Sensitivity & 1.00 & 0.96 & 0.85 & 0.94 & 0.80 & 0.90 & 0.97 & 0.80 \\
Specificity & 1.00 & 0.99 & 0.99 & 0.99 & 1.00 & 0.98 & 0.97 \\
Precision & 1.00 & 0.89 & 0.92 & 0.94 & 0.95 & 0.84 & 0.82 & 0.99 \\
Prevalence & 0.18 & 0.11 & 0.11 & 0.14 & 0.10 & 0.12 & 0.12 & 0.13 \\
\hline
\end{tabular}

Sensitivity: 'Correctly assigned' divided by the size of class; Specificity: 'Correctly rejected' divided by the sum of 'correctly rejected' and 'incorrectly assigned'; Precision: 'Correctly assigned' divided by the sum of 'correctly assigned' and 'incorrectly assigned'; Prevalence: Percentage of the class within the sample.

correctly. The algorithm takes the model which yields the highest tolerance for classification. Table 2 shows the obtained results if the algorithm uses the features identified as important in just a single trial: $97.2 \%$ of the spectral data are addressed correctly. The differences and commonalities allow a distinct discrimination of the bacteria as $100 \%$ will be assigned correctly if the final model is chosen after five trials. As the tuning parameter for the algorithm, the number of investigated models (trials) has been optimized to be at least 20. Fewer attempts weaken the validity and may include too many models with wrong decisions ('overfitting').

Now the remaining dataset is used to test the fitted model and its performance. The results of the classification are visualized in Table 3. In this test, e.g. the 42 spectra of B. atrophaeus are $100 \%$ correctly identified as $B$. atrophaeus, while the 26 samples of $B$. subtilis are identified as B. subtilis (84.6\%), B. brevis, E. coli, M. luteus and $P$. polymyxa (each $3.8 \%$ ). It is remarkable that there are highly correlated bacteria (according to Table 1) which are not assigned pairwise, and that other less correlated samples are misclassified. The corresponding performance parameters are given in Table 4. For this sample set the accuracy of the model is $91 \%$ (ratio of correctly identified and the total number).

\section{Summary and outlook}

In this paper, we have discussed standoff detection and classification prospects for bacteria using eight non-hazardous species as simulants for highly pathogenic bacteria. The LIF standoff spectra of very distant relatives as well as of closely related species have been shown for two different UV excitation wavelengths and classified using the C5.0 machine learning algorithm. Spectral diversity between the investigated eight bacterial species, among three species of the genus Bacillus, was analyzed and in a first attempt the different bacteria could be discriminated with an accuracy of better than $90 \%$ and a specificity of above $97 \%$. Further optimization of the discrimination algorithm by embedding decision tree pruning, principle component analysis and feature selection is expected to improve the identification performance.

A correlation analysis of the different spectra shows a strong relationship between the spectra for all sample combinations at $\lambda_{0}=355 \mathrm{~nm}$ and for some at $\lambda_{0}=280 \mathrm{~nm}$. The strong correlation of the spectra is a challenge for discrimination of bacteria. But the spectra obtained by excitation at a wavelength of $280 \mathrm{~nm}$ reveal the potential of LIF when exciting with an additional wavelength. 
It is promising that a third excitation wavelength below $270 \mathrm{~nm}$ in the absorption band of phenylalanine (a fluorophore present in bacteria) will provide further important information for the classification process and will be incorporated in the setup in the future. This experimental setup will also be useful for a broader spectrum of bacterial species. With a grown base of LIF data obtained with substantial numbers of samples in different concentrations and aggregation states, the distinction and classification of the samples will become more challenging. The method of cultivation and the environment may influence the bacteria and the fluorophores within. As an example, the ratio and the concentration of oxidized NAD and its reduced form NADH varies. In addition, the strains of the order Bacillales tested (see Figure 2) form endospores under unfavorable conditions which are quite different from vegetative cells in respect to structure and fluorescence spectra [32]. Since the investigated bacterial samples were stored in PBS (without carbon source and other important nutrients), it is likely that they contained endospores. Additionally, B. thuringiensis forms crystal proteins during sporulation [33], which also might influence the fluorescence spectra. Ongoing examinations of the spectral changes during the different stages of development of diverse bacteria are performed in our institute at the moment to investigate the influence of these conditions on the spectral features.

As indicated in the previously reported classification procedure [3], for liquids in a small cuvette or condensed matter on a surface, additional features might be obtained by an analysis of the fluorescence temporal behavior. The fluorescence lifetime of the dominant fluorophores in bacteria, e.g. tryptophan, other amino acids (tyrosine and phenylalanine) or nucleic acids, is on the order of below to a few nanoseconds and depends strongly on their microenvironment $[5,6]$. However, an expansion of the sample into an aerosol cloud, for example, influences the temporal behavior of the fluorescence signal. In such cases the measurement system needs to be adapted by, e.g. either a small overlap of the optical paths of detection and excitation or a simultaneous detection of the sample extent. Nevertheless, the measurement of the fluorescence lifetime may improve the classification process of biological samples and will be investigated in future experiments. For aerosols a polarization analysis of the scattered light may provide additional information.

In ongoing work, a more compact, transportable setup is set up for fluorescence detection, using shorter laser pulses for a more distinct temporal signature. It also uses a reduced number of detection channels, which will facilitate the data analysis and classification procedure. Training measurements are in progress and the results will be published.

Acknowledgment: F. Wilsenack, Bundeswehr Research Institute for Protective Technologies and CBRN Protection, Munster, Germany, is acknowledged for fruitful discussions on end user demands.

\section{References}

[1] D. Ivnitski, I. Abdel-Hamid, P. Atanasov and E. Wilkins, Biosens. Bioelectron. 14, 599-624 (1999).

[2] J. R. Simard, G. Roy, P. Mathieu, V. Larochelle, J. McFee and J. Ho, IEEE T. Geosci. Remote 42, 865-874 (2004).

[3] T. Fischbach, F. Duschek, A. Hausmann, C. Pargmann, V. Aleksejev, et al., Proc. SPIE 9455, 945508 (2015).

[4] Y. S. Cheng, Aerosol Sci. Tech. 30, 186-201 (1999).

[5] A. E. Kinkennon and L. B. McGown, J. Fluoresc. 7, 201-210 (1997).

[6] F. Awad, C. Ramprasath, N. Mathivanan, P. R. Aruna, and S. Ganesan, Biomed. Spectrosc. Imaging 3, 381-391 (2014).

[7] S. C. Hill, Y.-L. Pan, C. Williamson, J. L. Santarpia and H. H. Hill, Opt. Express 22, 22817-22819 (2014).

[8] L. Leblanc and É. Dufour, FEMS Microbiol. Lett. 211, 47-153 (2002).

[9] M. Sohn, D. S. Himmelsbach, F. E. Barton II and P. J. FeodorkaCray, Appl. Spectrosc. 63, 1251-1255 (2009).

[10] Y.-L. Pan, S. C. Hill, R. G. Pinnick, H. Huang, J. R. Bottiger, et al., Opt. Express 18, 12436-12457 (2010).

[11] Y.-L. Pan, S. C. Hill, R. G. Pinnick, J. M. House, R. C. Flagan, et al., Atmos. Environ. 45, 555-1563 (2011).

[12] V. Sivaprakasam, T. Pletcher, J. E. Tucker, A. L. Huston, J. McGinn, et al., Appl. Optics 48, B126-B136 (2009).

[13] V. Sivaprakasam, H.-B. Lin, A. L. Huston and J. D. Eversole, Opt. Express 19, 6191-6208 (2011).

[14] P. Jonsson, M. Elmqvist, O. Gustafsson, F. Kullander, R. Persson, et al., Proc SPIE 7484, 74840I (2009).

[15] M. Gelfusa, A. Murari, M. Lungaroni, A. Malizia, S. Parracino, et al., Proc. SPIE 9995, 99950W (2016).

[16] D. Joshi, D. Kumar, A. K. Maini and R. C. Sharma, Spectrochim. Acta A 112, 446-456 (2013).

[17] S. Buteau, J.-R. Simard, B. Déry, G. Roy, P. Lahaie, et al., Proc. SPIE 6378, 637813 (2006).

[18] S. Buteau, L. Stadnyk, S. Rowsell, J.-R. Simard, J. Ho, et al., Proc. SPIE 6756, 675608 (2007).

[19] S. Buteau and S. Rowsell, Proc. SPIE 9995, 99950B (2016).

[20] A. Hausmann, F. Duschek, T. Fischbach, C. Pargmann, V. Aleksejev, et al., Proc. SPIE, 9073, $90730 Z$ (2014).

[21] T. Fischbach, V. Aleksejev, F. Duschek, A. Hausmann, C. Pargmann, et al., 9th Future Security Conference Proceedings (Fraunhofer Verlag, Berlin, Germany, 2014) pp. 327-334.

[22] F. Duschek, T. Fischbach, A. Hausmann, C. Pargmann, J. Thieser, et al., 10th Future Security Conference Proceedings (Fraunhofer Verlag, Stuttgart, Germany, 2015) pp. 97-104. 
[23] A. Fix and G. Ehret. Intracavity frequency mixing in pulsed optical parametric oscillators for the efficient generation of continuously tunable ultraviolet radiation. Appl. Phys. 67:331-338 (1998).

[24] A. Fix, M. Wirth, A. Meister, G. Ehret, M. Pesch, and D. Weidauer. Tunable ultraviolet optical parametric oscillator for differential absorption lidar measurements of tropospheric ozone. Appl. Phys. B 75:153-163 (2002).

[25] F. Steinbach, A. Fix, M. Wirth, and G. Ehret. Development and application of an airborne differential absorption lidar for the simultaneous measurement of ozone and water vapor in the tropopause region. In Reviewed and revised paperspresented at the 26th international laser radar conference (ILRC 2012), volume 2, pp 1003-1006 (2012).

[26] H. Yi, Y.-J. Cho, D. Yong and J. Chun, J. Bacteriol. 194, 3742-3743 (2012).

[27] R Development Core Team, 'R: A Language and Environment for Statistical Computing' (Vienna, Austria: R Foundation for Statistical Computing, 2008).

[28] C. Spearman, Am. J. Psychol. 15, 72-101 (1904).

[29] J. Ross Quinlan, ‘C4.5: Programs for Machine Learning' (San Francisco, CA: Morgan Kaufmann Publishers, 1993).

[30] R Core Team, 'R: A Language and Environment for Statistical Computing', (Vienna, Austria: R Foundation for Statistical Computing, 2016).

[31] B. Lantz, 'Machine Learning with R' (Packt Publishing, Ltd., Birmingham, UK, 2nd edition, 2015).

[32] S. C. Hill, R. G. Pinnick, S. Niles, Y.-L. Pan, S. Holler, et al., Field Anal. Chem. Tech. 3, 221-239 (1999).

[33] N. Galitsky, V. Cody, A. Wojtczak, D. Ghosh, J. R. Luft, et al., Acta Crystallogr. D 57, 1101-1109 (2001).

\section{Frank Duschek}

German Aerospace Center

Institute of Technical Physics

Langer Grund Lampoldshausen

74239 Hardthausen, Germany

frank.duschek@dlr.de

After finishing his PhD on Resonance Raman Spectroscopy in 1996 at the University of Würzburg, Germany, Frank Duschek joined the Germany Aerospace Center (DLR) for research and development on high power and chemical lasers. Since 2008 , his main research interest is focused on standoff detection of chemical, biological and explosive hazards applying laser based spectroscopy. He is head of the department Atmospheric Propagation and Effect at the Institute of Technical Physics at DLR.

\section{Lea Fellner}

German Aerospace Center

Institute of Technical Physics

Langer Grund Lampoldshausen

74239 Hardthausen, Germany

Lea Fellner studied biology at the University of Osnabrück. Her doctoral thesis at the Technical University of Munich was concerned with investigation of overlapping genes of enterohemorrhagic $E$. coli (EHEC) and genome re-sequencing und re-annotation of an EHEC strain. Currently, she is head of the microbiological laboratory (S2) at the Institute of Technical Physics at the DLR Lampoldshausen and works on the standoff detection of biological agents.

\section{Florian Gebert}

German Aerospace Center

Institute of Technical Physics

Langer Grund Lampoldshausen

74239 Hardthausen

Germany

Florian Gebert studied physics at the Technical University Braunschweig, Germany, and graduated in 2009. Then, he joined the Quest Institute for Experimental Quantum Metrology at the German Metrology Institute in Braunschweig and received his PhD from the Leibnitz University of Hannover, for his work on precision isotope shift measurements of trapped $\mathrm{Ca}^{+}$ions, in 2015. Currently, he works at the German Aerospace Center (DLR) Lampoldshausen on laser-based standoff detection techniques.

\section{Karin Grünewald}

German Aerospace Center

Institute of Technical Physics

Langer Grund Lampoldshausen

74239 Hardthausen

Germany

Karin Grünewald studied Aerospace Engineering at the University of Stuttgart. She wrote her doctoral thesis on the Modelling of Energy Transfer Processes in Longitudinally Flown $\mathrm{CO}_{2}$ lasers. In 1986, she started her work in the R\&D of gas flow lasers at the Institute of Laser Technologies in Stuttgart. In 1994, she changed to chemical laser development at the DLR Institute of Technical Physics. After initial investigations on the atmospheric conditions and their effects on low energy laser propagation at the open space laser test range at DLR Lampoldshausen. She developed the framework for establishing the microbiological branch in the CBE detection and has been working on the optical characterization of bio-agents since 2012.

\section{Anja Köhntopp}

German Aerospace Center

Institute of Technical Physics

70569 Stuttgart

Germany

Anja Köhntopp received a Diploma in chemistry from Kiel University, Germany. In 2015, she finished her PhD on the ultrafast spectroscopy of functionalised gold nanoparticles at the Institute of Physical Chemistry in Kiel. She currently works on the preparation of concepts and studies concerning laser-based standoff detection of CBE substances at the Institute of Technical Physics at DLR.

\section{Marian Kraus}

German Aerospace Center

Institute of Technical Physics

Langer Grund Lampoldshausen

74239 Hardthausen

Germany

Marian Kraus studied Mathematics at the University of Heidelberg, Germany, where he wrote his diploma thesis about statistical methods of analysis and visualization of heart rate variability (HRV). Now he is employed at the German Aerospace Center (DLR) as a data analyst, emphasised by his passion for logic puzzles (including participation at the World Puzzle Championship). 


\section{Peter Mahnke}

German Aerospace Center

Institute of Technical Physics

70569 Stuttgart, Germany

Peter Mahnke studied physics at the Technical University of Kaiserslautern, Germany. Since 2001, he has been working at the Germany Aerospace Center Stuttgart, developing laser and nonlinear optics sources for remote sensing applications. For a helicopter based methane differential absorption lidar (DIAL) he developed a $3.2 \mu \mathrm{m}$ injection seeded OPO-laser source. The system received the DLR Innovation Award 2006. Furthermore, he developed laser sources from the mid infrared to the UV for amongst others water vapor DIAL, laser-ultrasound excitation in carbon fiber plastics, and UV fluorescence excitation of formaldehyde and biological agents.

\section{Carsten Pargmann}

German Aerospace Center

Institute of Technical Physics

Langer Grund Lampoldshausen

74239 Hardthausen, Germany

Carsten Pargmann finished his PhD in the field of low temperature plasma physics at Ruhr-University Bochum in 2006. Since then he has been working at the German Aerospace Center (DLR) Lampoldshausen on the design of resonators and optical spectroscopy systems and the standoff detection of hazardous materials.

\section{Herbert Tomaso}

Friedrich-Loeffler-Institute

Institute of Bacterial Infections and Zoonoses

07743 Jena, Germany

Herbert Tomaso studied human medicine at the Karl-Franzens

University in Graz (Austria). He wrote his doctoral thesis about the
AIDS epidemic in Austria and was promoted in 1995. In the same year he obtained a diploma in tropical medicine and parasitology at the Bernhard-Nocht Institute in Hamburg (Germany). After the specialization as a general practitioner (1998) and obligatory military training he started his specialization in medical microbiology at the Medical University in Innsbruck (Austria). From 2002 until 2008 he worked on the detection of bacterial agents at the Bundeswehr Institute of Microbiology in Munich (Germany) as a liaison officer of the Austrian Armed Forces. He wrote his habilitation treatise about the detection of bacterial agents using real-time PCR techniques and obtained the venia docendi in medical microbiology at the Medical University Graz in 2008. Since September 2008, he has worked in the Friedrich-Loeffler Institut, which is the Federal Research Institute for Animal Health. He is deputy director of the Institute of Bacterial Infections and Zoonoses and head of the National Reference Laboratory for Tularemia.

\section{Arne Walter}

German Aerospace Center Institute of Technical Physics Langer Grund Lampoldshausen 74239 Hardthausen, Germany

Arne Walter graduated from Georg-August-University, Göttingen, Germany, in 2009 with a Diploma in Chemistry and received his $\mathrm{PhD}$ in Chemistry in 2014. Previous work includes ultrafast laser spectroscopy of fluorescent proteins at the Max-Planck-Institute for Biophysical Chemistry in Göttingen. Presently, his main research area at the German Aerospace Center Lampoldshausen is standoff detection of biological and chemical agents with laser systems. 